ti, in particolare S. epidermidis, seguito da $S$. aureus; sono però presenti anche altri potenziali patogeni ( $P$. aeruginosa, E. faecalis, E. coli, M. morganii, Acinetobacter spp., Candida spp.) e anche microrganismi di raro riscontro come K. kristinae. B- L'emocoltura quantitativa e l'emocoltura basata sul DTP sono tecniche, nella nostra piccola casistica, di esecuzione abbastanza semplice e con una buona corrispondenza di risultato tra loro (20 casi studiati di cui 3 positivi).

Conclusioni: A- 1- La Tecnica di Cleri è una Tecnica molto valida e pratica; tenere abbinata a questa la tecnica qualitativa consente di aumentare ulteriormente la sensibilità, già buona, della sola Tecnica di Cleri; 2- Gli Stafilococchi sono i microrganismi più frequentemente isolati. Possono però essere coinvolti anche altri potenziali patogeni e anche microrganismi di raro riscontro; B- L'emocoltura quantitativa e l'emocoltura basata sul DTP sono tecniche abbastanza semplici e molto utili per la diagnostica microbiologica delle infezioni CVC associate; tenendo presenti pregi e limiti dell'una e dell'altra metodica l'utilizzo contemporaneo di entrambe risulta utile e più completo.

\title{
051
}

\section{DIAGNOSTICA MICROBIOLOGICA DELLE INFEZIONI ASSOCIATE AI CVC: STUDIO IN PAZIENTI IMMUNOCOMPROMESSI.}

\author{
Basaglia G., Stocco Calzavara S., Pancino A., Sperandio P., \\ Tomasini M.L., De Paoli P. \\ S.C. Microbiologia, Immunologia e Virologia, CRO, \\ Istituto Nazionale Tumori-IRCCS, Aviano (Pordenone).
}

Introduzione: L'utilizzo di cateteri vascolari è oggi una pratica sempre più diffusa con diverse applicazioni cliniche. Una delle complicanze più importanti di tale pratica sono le infezioni. Scopi del nostro lavoro sono stati, con riferimento ai pazienti seguiti presso il nostro Centro (pazienti oncologici, ematologici e con AIDS, frequentemente anche immunocompromessi, nei quali la complicanza infettiva associata al cateterismo vascolare può assumere significati di particolare gravità): A- Riesame dei dati della casistica pluriennale del nostro Laboratorio relativi alla diagnostica microbiologica delle infezioni CVC associate; B- Allestimento dell'emocoltura quantitativa e dell'emocoltura basata sul "differential time to positivity" (DTP) approfondendone pregi e limiti.

Metodi: Emocoltura qualitativa ed Emocoltura basata sul DTP (Sistema automatico Bactec 9120, Becton Dickinson), Emocoltura quantitativa (Sistema Isolator, Oxoid), Esame colturale qualitativo ed esame colturale quantitativo secondo Cleri della punta del CVC.

Risultati: A- In caso di risultato significativo con la Tecnica di Cleri le emocolture sono positive nel $71.2 \%$; la Tecnica qualitativa ha invece percentuali di falsi positivi che vanno dal $66.7 \%$ al $75 \%$; solo in pochi casi con risultato non significativo o negativo con la Tecnica di Cleri, ma positivi con la Tecnica qualitativa, le emocolture sono risultate positive. Gli Stafilococchi sono i microrganismi più frequentemente isola- 